\title{
Pars plana vitrectomy in the management of pupillary block glaucoma following irrigation and aspiration
}

\author{
GHOLAM A. PEYMAN, DONALD R. SANDERS, AND HARVEY MINATOYA \\ From the Department of Ophthalmology, University of Illinois Eye and Ear Infirmary, Chicago, USA
}

SUMMARY Pupillary block glaucoma developed in 3 children treated with irrigation and aspiration using the Cavitron phacoemulsification system. One had a congenital cataract, 1 had blunt trauma, and 1 had a penetrating injury. The factors contributing to pupillary block included excessive retained lens material, severe post-traumatic inflammation with synechia formation in 2 cases, and the lack of iridectomy in a case requiring a membrane discission. The pupillary block was successfully treated by pars plana vitrectomy in all cases; however, vision in 1 eye was lost owing to secondary glaucoma resulting from delay in treatment and the development of $360^{\circ}$ peripheral anterior synechiae.

Aphakic pupillary block glaucoma occurs when aqueous flow from the posterior to the anterior chamber is obstructed. This obstruction may result from postoperative wound leak or inflammation after either intracapsular or extracapsular lens extraction (Sugar, 1966; Cotlier, 1972). We performed pars plana vitrectomy in 3 patients to treat pupillary block that occurred after irrigation and aspiration with the Cavitron phacoemulsification unit.

\section{Case reports}

\section{CASE 1}

A 10-year-old boy had undergone phacoemulsification to remove a dense, nuclear congenital cataract in the right eye on 29 July 1975. No iridectomy was done, and at the end of the procedure a dense opacity on the intact posterior capsule was present and was resistant to all attempts at removal.

On 6 October 1975 the patient had visual acuity of hand motions. Intraocular pressure by applanation tonometry was $18 \mathrm{mmHg}$ in the right eye, and a dense secondary membrane precluded fundus visualisation. On 7 October with the patient under general anaesthesia a Sato knife discission of the membrane was performed without complication.

The patient was referred to us for treatment on 28 October 1975 with a 3-week history of raised intraocular pressure and a presumed aphakic

Address for reprints: Dr Gholam A. Peyman, University of Illinois Eye and Ear Infirmary, 1855 W. Taylor Street, Chicago, Illinois 60612, USA pupillary block glaucoma. His visual acuity was counting fingers at $1 \mathrm{ft}(30 \mathrm{~cm})$ with a $+12 \cdot 50$ sphere. Conjunctival examination showed $2+$ injection, and the cornea exhibited $2+$ epithelial oedema without keratic precipitates. The anterior chamber was of aphakic depth centrally with the vitreous face mushrooming forward and filling $95 \%$ of the anterior chamber. Gonioscopy showed $360^{\circ}$ of peripheral anterior synechia; no angle structure was visible. Intraocular pressure was $46 \mathrm{mmHg}$. Fundus findings were normal. On 29 October a vitrectomy, sector iridectomy, and membranectomy were performed with the vitrophage through a $3-\mathrm{mm}$ limbus-parallel pars plana incision. There were no operative complications.

During the first postoperative week corneal oedema and minimal anterior chamber reaction began to clear; the intraocular pressure varied between 21 and $29 \mathrm{mmHg}$ with the patient receiving atropine and Maxitrol (polymyxin B, neomycin, dexamethasone) drops. The view of the posterior fundus was consistent with 20/30 to 20/40 vision. The patient was discharged on a regimen of acetazolamide $125 \mathrm{mg} 4$ times a day, and atropine and Maxitrol drops 3 times a day. Two weeks postoperatively the cornea was clear and intraocular pressure was $15 \mathrm{mmHg}$.

Unfortunately the patient's ocular pressure became uncontrollable, and an ophthalmologist's report indicated that his vision was reduced to no light perception 6 months after surgery.

CASE 2

A 7-year-old girl had a history of right esotropia of 
congenital onset that had been treated with glasses at the age of 5 years. On 1 May 1977 she was playing without her glasses and was struck in the right eye by a rock. On presentation her uncorrected visual acuity was $20 / 200$ in the right eye and $20 / 50$ in the left eye. A variable right esotropia was noted. The conjunctiva was mildly hyperaemic and a corneal abrasion was present. The anterior chamber of the right eye was deep and showed a $4+$ flare and cells. The cells were red blood cells and no layering was seen. There were multiple tears in the iris sphincter. A diagonal rent was visible in the superotemporal periphery of the anterior capsule; otherwise the lens was clear. The fundus showed diffuse patches of Berlin's oedema. The left eye was normal. In hospital the hyphaema gradually cleared. A slight bulging forward of the lens cortex was noted at the site of the rent. This stabilised and the lens did not opacify in this area; a trace posterior subcapsular flower did appear, however. The patient was discharged on the fifth hospital day on a regimen of homatropine drops 4 times a day. The anterior chamber reaction continued to clear, but the lens progressively opacified.

On 17 May 1977 she was readmitted with a visual acuity best corrected to $20 / 400$ in the right eye and $20 / 25$ in the left eye. A $40^{\circ}$ right esotropia was noted. The anterior chamber of the right eye showed $1+$ flare and rare cells. The lens showed $3+$ anterior cortical and $2+$ posterior subcapsular changes. The fundus showed minimal residual Berlin's oedema. Applanation tension was $14 \mathrm{mmHg}$ in both eyes. The next day irrigation and aspiration of the cataract in the right eye was performed through an incision made at 11 o'clock. An 'O' capsulotomy was made, and the Kelman irrigation and aspiration tip was used. A peripheral iridectomy was performed and the wound was closed with two 8.0 polyglactin 910 sutures. Postoperative medication consisted of $1 \%$ atropine and topically applied Neosporin (polymyxin B, neomycin, gramicidin) 4 times a day. On the first postoperative day a small amount of residual cortex was apparent in the capsular bag in the superotemporal quadrant. A slow trickle of blood was seen originating at the 12 o'clock site of a previous posterior synechia. The next day a tiny clot remained at the pupillary margin, and the anterior chamber reaction showed $2+$ flare and cells. By the fourth postoperative day the blood was gone and only $1+$ flare and cells were noted. A few spider-web-like strands of fibrin remained on the intact posterior capsule. She was discharged on a regimen of atropine 4 times a day, which maintained dilatation of the pupil at $7 \mathrm{~mm}$.

One week postoperatively it was noted that the posterior capsule had cleared but had moved anteriorly into contact with the iris. A few areas of posterior synechiae were forming. Applanation tension was $10 \mathrm{mmHg}$. She was given $10 \%$ phenylephrine hydrochloride 4 times a day in addition to the atropine. Two weeks postoperatively the anterior chamber showed $1+$ flare and occasional cells. The pupil was dilated to $8 \mathrm{~mm}$, but more posterior synechiae had formed. Inspection disclosed the peripheral iridectomy to be narrowed by the dilatation and the rim of anterior capsule to be in close approximation to the iris. Applanation tension was $12 \mathrm{mmHg}$.

On 10 June 1977 she had complaints of pain in the right eye for 3 days' duration. Examination showed epithelial corneal oedema. The anterior chamber was deep centrally but closed peripherally (Fig. 1). The pupil had become secluded $360^{\circ}$ and iris bombé was present. A $2+$ flare and $2+$ cellular reaction was noted. The peripheral iridectomy was also secluded by anterior capsule. Applanation tension was $70 \mathrm{mmHg}$. The fundus appeared normal. She was readmitted and given intravenous mannitol. That same day an anterior chamber paracentesis was done first to partially decompress the globe. Then an inferotemporal sclerotomy was made and a para plana capsulectomy and vitrectomy was performed using the Peyman vitrophage. The posterior capsule was removed, and a spontaneous deepening of the anterior chamber was noted. A generous anterior vitrectomy was then performed. Interestingly, after decompression and before

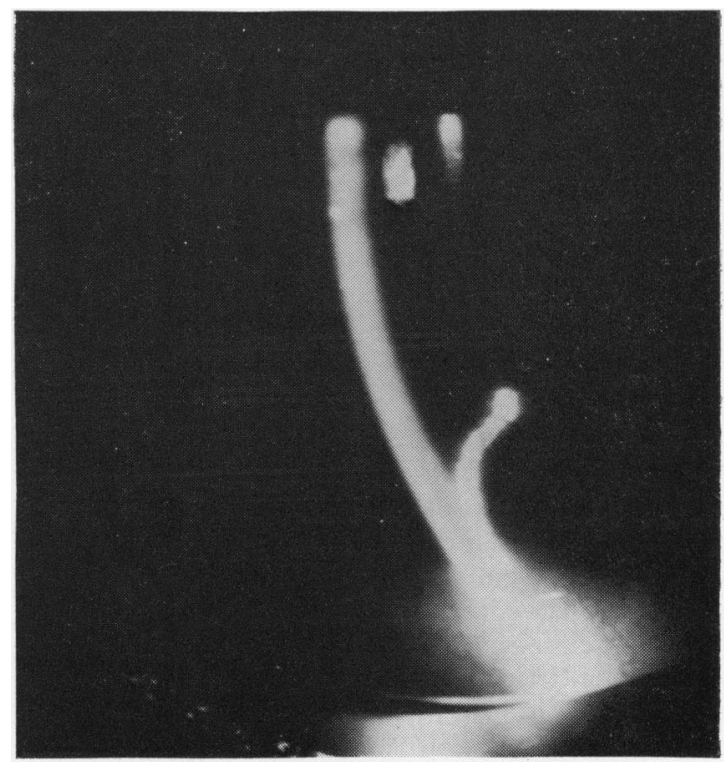

Fig. 1 Case 2. Preoperative slit-lamp photograph demonstrating iris bombé inferiorly 


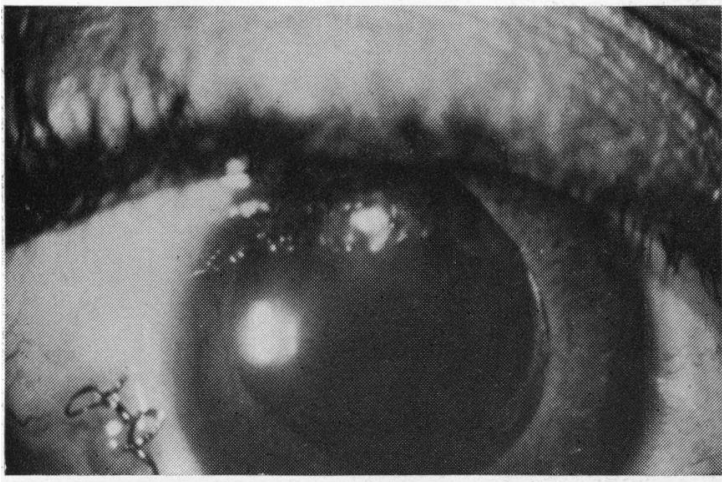

a

proceeding with the vitrectomy, examination of the fundus revealed dilated veins with scattered flameshaped retinal haemorrhages.

In the postoperative period, the anterior chamber remained of aphakic depth with $2+$ flare and cells. The applanation tension was $12 \mathrm{mmHg}$. The patient was discharged on the fourth postoperative day on a regimen of atropine and Maxitrol 4 times a day. On follow-up visits, the anterior chamber reaction and the retinal flame-shaped haemorrhages slowly cleared. The intraocular tension has remained normal, and the patient has 20/50-3(6/15) vision with an aphakic contact lens 5 months postoperatively (Fig. $2 a, b$ ).

CASE 3

A 6-year-old boy was seen on 27 February 1977 6 hours after being hit in the left eye with a rock. Ocular examination disclosed an $8 \mathrm{~mm}$ full-thickness inferior corneal laceration and a partially formed anterior chamber with iris incarceration. Centrally there appeared to be a fibrin clot in the anterior chamber. The lens could be seen only poorly through the clot, but it was not grossly cataractous. The posterior fundus could not be visualised. The patient was operated on by repairing his corneal laceration with seven 10.0 nylon sutures.

Postoperatively the corneal wound appeared to heal well. The anterior chamber was well formed and an inferior bulge was noted over the lens, which was interpreted as either a fibrinous membrane or a rent in the lens capsule. No flocculent lens material was noted in the anterior chamber. The lens became progressively opaque, and on 1 April 1977 the patient underwent an irrigation and aspiration of his cataract. An ' $O$ ' capsulotomy was made and the Kelman irrigation and aspiration tip was used. A peripheral iridectomy was performed, and the 3 $\mathrm{mm}$ superior incision was closed with two 8.0 polyglactin 910 sutures. Postoperative medication included atropine and Maxitrol ointments. On the

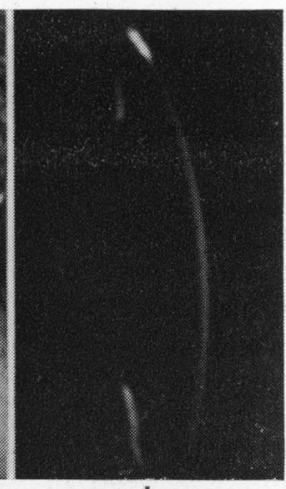

Fig. 2a Case 2. Postoperative anterior segment photograph demonstrating clear cornea. b. Slit-lamp photograph demonstrating deep anterior chamber and relief of pupillary block

first postoperative day the conjunctiva was mildly hyperaemic, the cornea was clear, and the anterior chamber was deep, although a somewhat hazy view of the iris was noted. The red reflex was dull. Atropine and Maxitrol treatment was continued, and on the fourth postoperative day diffuse corneal epithelial oedema appeared and the eye seemed hard to tactile pressure. (The patient was uncooperative for further intraocular pressure testing.) Adrenaline $2 \%$ twice a day and acetazolamide 125 mg every 6 hours were instituted. On the seventh postoperative day the conjunctiva was mildly hyperaemic, the cornea was clear except for the inferior scar, the anterior chamber was deep with minimal reaction, the pupil was $4 \mathrm{~mm}$ and round, and a $4+$ red reflex was present. The patient was discharged on a regimen of atropine ointment twice a day and Maxitrol ointment 4 times a day.

On the eleventh postoperative day increased redness and photophobia with advanced conjunctival hyperaemia were noted; tactile ocular pressure appeared normal. The Maxitrol ointment was increased to every 2 hours.

On the fifteenth postoperative day a Van Lint block was given for a more complete examination because the child was unco-operative. There was an increase in conjunctival reaction, the cornea was clear, tactile and Schiotz pressures were grossly normal, and the anterior chamber was deep. A short course of oral steroids was added to the topical regimen.

On the twenty-first postoperative day the conjunctiva had $2+$ hyperaemia, the cornea showed epithelial oedema, the anterior chamber was very shallow with iris thought to be touching cornea in the periphery, and a $1+$ cellular reaction was seen. The applanation tension was approximately 34 $\mathrm{mmHg}$. Acetazolamide $125 \mathrm{mg}$ was given every 6 hours and further mydriasis was attempted. On the twenty-fifth postoperative day the patient had frank iris bombé with a totally secluded pupil. The iris 
was touching the cornea peripherally for $360^{\circ}$. A pars plana capsulectomy and anterior vitrectomy were performed with the Peyman vitrophage. When the posterior capsule was removed, the anterior chamber deepened spontaneously to aphakic depth. Iris bleeding was noted when the vitrophage tip merely touched the iris margin, so an iridectomy was not performed. Postoperatively the patient was given atropine ointment twice a day, Maxitrol ointment 4 times a day, and acetazolamide $125 \mathrm{mg}$ every 6 hours.

On the third postoperative day an examination with the patient under anaesthesia revealed conjunctival hyperaemia, an $8 \mathrm{~mm}$ vascularised corneal scar with an otherwise clear cornea, an anterior chamber of aphakic depth, and Schiotz tension of 10 scale units with a $5.5 \mathrm{~g}$ weight. A clear view to the posterior fundus was seen with an indirect ophthalmoscope.

The postoperative course after 9 months of followup with no medication has been uneventful. Applanation tensions have varied between 18 and $25 \mathrm{mmHg}$. The patient has a $35^{\circ}$ left exotropia, and refracts with trial lenses to $20 / 100-1$ with a $+9 \cdot 25$ sphere +5.50 cylinder $\times 45^{\circ}$ axis. The patient is at present being evaluated for a contact lens to correct the irregular astigmatism produced by the corneal scar.

\section{Discussion}

Aphakic pupillary block glaucoma occurs most commonly after cataract surgery complicated by postoperative wound leak, severe postoperative inflammation, or both. With uncomplicated irrigation and aspiration or phacoemulsification the small incision makes wound leak unlikely and inflammation minimal. Thus pupillary block is usually a rare complication with these procedures.
We believe that complicating factors in our cases included excessive amount of inflammation as a result of retained lens material (Cases 1, 2, and 3), as a result of previous trauma (Cases 2 and 3), and because an iridectomy was not performed which contributed to pupillary block following a membrane discission (Case 1).

In the past, if medical management of aphakic pupillary block was unsuccessful, peripheral iridectomy was suggested. If the anterior chamber did not spontaneously reform, fluid was aspirated with a needle from the retrovitreal space and the anterior hyaloid face was slashed.

Unfortunately, past surgical treatment did not permanently eliminate the factors that produce pupillary block. Thus we think that procedures using an automated vitreous instrument such as pars plana removal of retrovitreal or iridocapsular adhesions, iridectomy, vitrectomy, and membranectomy may be beneficial.

The timing of operation is of critical importance. In Case 1 the patient was treated medically with closed angles for 3 weeks, and visual acuity progressed to no light perception because of uncontrolled glaucoma after the pupillary block was relieved. In the other 2 cases pupillary block with closed angles was present for less than 6 days, and the pressure returned to normal after treatment.

This research was supported in part by a grant from the Illinois Lions Foundation.

\section{References}

Sugar, H. S. (1966). Pupillary block and pupillary-block glaucoma: following cataract extraction. American Journal of Ophthalmology, 61, 435-443.

Cotlier, E. (1972). Anterior vitrectomy for aphakic flat anterior chamber. British Journal of Ophthalmology, 56, 347-352. 